\title{
Philosophiques
}

\section{Michael Friedman, Dynamics of Reason, Stanford, CSLI Publications, 2001, 141 pages.}

\section{Yvon Gauthier}

Volume 31, numéro 1, printemps 2004

Poincaré et la théorie de la connaissance

URI : https://id.erudit.org/iderudit/008951ar

DOI : https://doi.org/10.7202/008951ar

Aller au sommaire du numéro

Éditeur(s)

Société de philosophie du Québec

ISSN

0316-2923 (imprimé)

1492-1391 (numérique)

Découvrir la revue

Citer ce compte rendu

Gauthier, Y. (2004). Compte rendu de [Michael Friedman, Dynamics of Reason, Stanford, CSLI Publications, 2001, 141 pages.] Philosophiques, 31(1), 269-271.

https://doi.org/10.7202/008951ar d'utilisation que vous pouvez consulter en ligne.

https://apropos.erudit.org/fr/usagers/politique-dutilisation/ 
sciences (cf. Ramsey, Logique, philosophie et probabilités, op. cit., p. 252). Nous sommes certes loin des préoccupations actuelles quant à la question de la complexité. Mais il est clair que la théorie de 1926-1928 de Ramsey, tout comme la récente "sémantique du succès ", a été élaborée sans prendre en considération ces problèmes; elles participent, si l'on veut, du paradigme de la rationalité «instrumentale». Ramsey avait lui-même ouvert la porte à la remise en cause des idéalisations fortes (telles que l'omniscience dénoncée par l'objection de la surcharge cognitive ou, pour ne prendre qu'un autre exemple, la révision des croyances par "conditionalisation» — qui n'a cependant pas de lien avec le soidisant «test de Ramsey» - , qui est une fonction à croissance exponentielle et qui n'est donc pas «faisable» en pratique) que comportent sa théorie de 1926-1928 et celles qui s'en inspirent; idéalisations qui expliquent en partie les difficultés rencontrées lorsqu'on veut les appliquer à des cas concrets. N'écrivait-il pas dès "Vérité et probabilité»: "On pourrait dire qu'on devrait penser ce qui est vrai, mais en ce sens là [...] on ne peut non plus trouver quelque justification de la croyance partielle; la chose idéale serait d'avoir des croyances de degré 1 en toute proposition vraie et de degré 0 en toute proposition fausse. Mais c'est trop demander à des hommes mortels, et on doit convenir qu'un certain degré de doute, ou même d'erreur, est humainement justifié» (Ramsey, Logique, philosophie et probabilités, op. cit., p. 176). On est donc en droit d'espérer que ces dernières idées sauront inspirer de nouveaux développements. En attendant, il faut remercier les auteurs pour avoir mis à notre disposition une introduction non seulement à l'œuvre de Ramsey mais aussi à une théorie contemporaine qui en revendique l'héritage.

MATHIEU MARION

Université du Québec à Montréal

\section{Michael Friedman, Dynamics of Reason, Stanford, CSLI Publications, 2001, 141 pages.}

Ce recueil est constitué des conférences sur Kant que l'auteur a prononcées en 1994 à l'Université Stanford dans le cadre d'un cycle de Kant Lectures. Il s'agit moins d'un ensemble d'études sur Kant que d'une tentative de récupérer l'héritage kantien et de le confronter au legs du positivisme logique. Le premier chapitre est en effet consacré à l'idée d'une philosophie scientifique, idée chère à un Hans Reichenbach. Mais c'est surtout l'empirisme logique de Carnap que Friedman veut sauvegarder face au naturalisme prôné par un Quine qui n'aurait pas réussi à prendre la mesure de l'entreprise carnapienne. C'est aussi à un Kuhn reconduit à ses sources rationalistes et contre le relativisme post-kuhnien que nous convie Friedman, qui voudrait dynamiser et en même temps historiciser l'a priori kantien.

L'auteur connaît bien Kant et ses sources scientifiques; son Kant and the Exact Sciences (1992) montre comment Kant se serait laissé entraîner sur la pente glissante de la déduction transcendantale d'une physique (et d'une chimie) fondée sur des principes a priori. L'Opus Postumum esquisse une théorie du "Wärmestoff" ou éther calorique qui voudrait faire le pont entre le transcendantal et l'empirique. On sait que Hegel a voulu remonter cette pente dans une physico-chimie dénaturée, 
et l'auteur n'a pas voulu commenter la suite. Friedman est aussi un commentateur compétent de la théorie de la relativité et son ouvrage de 1985, Foundations of Space-Time Theories: Relativistic Physics and the Philosophy of Science, aurait pu servir de prétexte à la rencontre avec Kant et les principes constitutifs, comme Friedman préfère les appeler dans sa discussion des fondements de la physique einsteinienne aussi bien que dans la défense de la théorie carnapienne des cadres linguistiques "linguistic frameworks»(p. 32 et sq.).

Les deuxième et troisième chapitres portent sur les perspectives historiques du clivage entre science et philosophie et entre paradigmes kuhniens et rationalité habermassienne. Il y a chez notre auteur une volonté de rassemblement œcuménique, et on ne peut que se réjouir d'une entreprise qui vise à la réconciliation de la science et de la philosophie dans une philosophie des sciences ouverte et animée par la dynamique de la raison.

La deuxième partie de l'ouvrage (p. 71-129) est justement consacrée à une discussion ouverte sur les thèmes des conférences qui occupaient la première partie. Friedman semble défendre au fil de la discussion avec ses nombreux interlocuteurs une sorte de constructivisme mitigé, comme on dit réalisme mitigé. L'étiquette n'est pas gênante pour l'auteur puisqu'il avoue que sa défense des principes constitutifs de la rationalité scientifique s'accorde très bien avec l'empirisme constructif d'un van Fraassen, par exemple (p. 84).

On peut se demander si cette version affaiblie du constructivisme rend justice à la notion de principe constitutif. On en voudra pour exemple le principe d'équivalence dans la théorie einsteinienne de la relativité générale, dont Friedman nous dit qu'il n'était qu'une possibilité mathématique avant de devenir, par l'entremise d'Einstein, une possibilité empirique - physique ou réelle - (p. 113). Un principe de coordination fondé sur l'équivalence entre masse inerte et masse gravitationnelle doit assurer le passage de la variété semi-riemannienne de la géométrie quadridimensionnelle de l'espace-temps à sa réalisation ou application empirique (p. 81). Là-dessus, Friedman rejette du revers de la main la remarque de H. Weyl (p. 113, note 54) qui suggérait que Riemann avait anticipé la relativité générale. Mais ce n'est pas ce que suggère $H$. Weyl, qui dit seulement que c'est le contenu physique qui détermine la configuration empirique de la structure topologique et géométrique. Mais il n'y a pas de passage direct de la structure abstraite à sa réalisation empirique par la médiation du principe d'équivalence comme semble le suggérer à son tour Friedman, qui passe sous silence le principe de Mach dans le présent ouvrage. Les équations du champ d'Einstein ne sont pas canoniques et ne génèrent pas qu'un seul modèle dont découlerait "naturellement " l'interprétation physique. (p. 64). L'importance toute relative accordée à l'appareil analytique ou au formalisme logico-mathématique invite à penser que l'auteur adopte une version édulcorée des principes constitutifs. S'il reconnaît le rôle prépondérant qu'a joué Poincaré dans la formation des idées d'Einstein, il néglige la dimension proprement constitutive du conventionnalisme de Poincaré qui voyait dans les "bonnes" conventions des constructions bien fondées. On notera enfin un apparent contresens (note 63, p. 123-124) sur la notion de cause commune qui rendrait compte des corrélations mises à jour par les inégalités de Bell en mécanique quantique. L'argument, tiré de Reichenbach, a été utilisé par van Fraassen pour montrer que les corrélations de spin pour deux particules spatialement éloignées l'une de l'autre ne pourraient s'expliquer par le recours à une cause commune. La méprise est significative puisqu'elle s'appuie sur une interprétation réaliste de la 
mécanique quantique et non sur l'interprétation standard de Bohr-Heisenberg ou «interprétation de Copenhague ", qui met plutôt l'accent sur les principes indéterministes constitutifs du formalisme quantique.

On ne chicanera pas l'auteur sur ces questions qui sont en réalité subordonnées à son propos principal, la réconciliation des traditions philosophiques du constructivisme kantien avec le positivisme carnapien et la dimension historiciste des révolutions scientifiques, ou mieux, de l'évolution dynamique des théories scientifiques. La connaissance précise des rapports de Kant avec la science de son temps aura permis à Friedman de proposer au philosophe contemporain un modèle de réflexion où science et philosophie entretiennent des liens constants dans une communication rationnelle (Habermas) qui convie tous les acteurs de la construction théorique du monde (Weyl).

YVON GAUTHIER

\section{Marc Ereshefsky, The Poverty of the Linnaean Hierarchy: A Philoso- phical Study of Biological Taxonomy, Cambridge, Cambridge University Press, 2001, 328 pages.}

Le livre de Marc Ereshefsky vise à la fois à initier ceux qui désirent découvrir les problématiques philosophiques inhérentes à la taxinomie biologique et à défendre certaines positions particulières sur celles-ci. La taxinomie biologique est une sousdiscipline de la biologie, dont l'objectif central est de déterminer les principes qui devraient guider la classification des entités vivantes. L'auteur développe au sein de son livre trois thèses concernant ces principes ainsi que le système de classification qu'ils motivent. Il argumente en premier lieu que seuls les schèmes de classification historiques conviennent à la taxinomie biologique. En second lieu, il défend une conception pluraliste de la classification taxinomique. Finalement, il soutient que le système taxinomique linnéen doit être abandonné au profit d'un ensemble de systèmes de classification qui n'attribuent pas de rang fixe aux taxons (i.e., aux unités taxinomiques telles que les espèces, les genres, etc.).

La première des trois parties de l'ouvrage cherche, d'une part, à introduire le lecteur aux diverses façons de concevoir la classification des entités (et, plus particulièrement, des taxons), et vise, d'autre part, à défendre une conception historique de la taxinomie biologique. Dans les deux premiers chapitres du livre, Ereshefsky décrit tour à tour les trois plus importantes conceptions de la classification des entités (l'essentialisme, les approches «faisceaux» (cluster), et l'approche historique), les principales écoles de taxinomie biologique (la taxinomie évolutionniste, le phénéticisme, le cladisme de Hennig, et le cladisme "transformé » de Nelson et Platnick), ainsi que les principales façons de concevoir la catégorie taxinomique, qui a sans doute reçu le plus d'attention de la part des biologistes évolutionnistes, soit l'espèce biologique (les conceptions biologique, écologique, phylogénétique, etc., d'espèce). Ce mode de présentation a l'avantage de permettre au lecteur de lier les diverses conceptions de la taxinomie biologique à des thèses beaucoup plus générales sur la classification des entités.

Dans le troisième chapitre du livre, Ereshefsky soutient que la conception essentialiste de la classification (laquelle exige que les entités classées ensemble partagent une même essence) et les approches «faisceaux» (lesquelles requièrent plutôt 